\title{
Societas Europaea Physiologiae Clinicae Respiratoriae
}

Chest Wall and Ventilatory Failure

Barcelona, Spain, June 11-15, 1984

Topics will include: (1) Structure and function of respiratory muscles and chest wall and (2) Applied physiology and diseases of the respiratory pump. Three symposia dealing with chest wall mechanics, muscle fatigue, and testing and therapeutics of the respiratory muscles will be organized on June 12, 13,

and 14, respectively. Papers and posters on the main subjects or free communications will be accepted before February 15, 1984 (limit for abstract submission). The 11th and 15th June will be devoted to Working Group meetings in parallel Sessions.

For further information please write to: OTAC SA, Chest Wall and Ventilatory Failure, Technical Secretariat, C/ Sepúlveda, 4547, Barcelona 15, Spain 